\title{
Adherence and Subthreshold Adherence in Sleep Apnea Subjects Receiving Positive Airway Pressure Therapy: A Retrospective Study Evaluating Differences in Adherence Versus Use
}

\author{
Barry Krakow MD, Victor A Ulibarri, Michelle R Foley-Shea, Alyssa Tidler, and \\ Natalia D McIver
}

\begin{abstract}
BACKGROUND: Research on subthreshold compliance with positive airway pressure (PAP) therapy in sleep apnea patients may inform clinical sleep medicine practice. We retrospectively assessed compliant and subthreshold compliant sleep apnea subjects to test whether regular but fewer hours of PAP use would demonstrate clinically meaningful improvements and correlate with outcomes. METHODS: A chart review was conducted of 113 consecutive sleep apnea subjects, naive to treatment, who completed a titration study and filled a PAP therapy prescription. Objective data categorized subjects into 3 groups: compliant, subthreshold compliant, and minimal use. Outcome measures assessed changes in insomnia, sleepiness, and nocturia on average 7 months from PAP initiation. Correlation coefficients analyzed dose-response relationships between hours of use and changes in outcomes. RESULTS: Among 113 PAP attempters, 104 (92\%) were current users. Among 93 users with objective data, regular (consistent) PAP users included 59 compliant and 21 subthreshold compliant, and 13 subjects were minimal users. Compliant users averaging $6.6 \pm 1.3 \mathrm{~h} / \mathrm{night}$ and $42.0 \pm 12.1 \mathrm{~h} /$ week showed the largest outcome improvements $($ all $P<.05)$ with moderate to large effects for insomnia $(d=0.94)$, sleepiness $(d=0.58)$, and nocturia $(d=0.56)$. Subthreshold users averaging $4.1 \pm 0.7 \mathrm{~h} / \mathrm{night}$ but only $18.0 \pm 5.6 \mathrm{~h} /$ week showed a large effect for insomnia $(\mathrm{d}=0.76, P=.03)$ and nonsignificant, small effects for sleepiness $(\mathrm{d}=0.38)$ and nocturia $(d=0.22)$. Correlation coefficients showed a trend for decrease in insomnia $(P=.08 ; r=.20)$ and a significant decrease in nocturia $(P=.034 ; r=0.25)$, each in association with hours of PAP use. CONCLUSIONS: In a clinical sample, $86 \%$ of sleep apnea subjects regularly used PAP, but adherence was $63 \%$. Regular users showed clinical treatment effects and potential dose-response relationships, suggesting that the term use offers advantages over the term adherence. Currently, subthreshold compliance may not merit insurance coverage in many countries, an issue affecting many sleep apnea patients. Key words: compliance; adherence; continuous positive airway pressure; obstructive sleep apnea; upper airway resistance syndrome; Centers of Medicare and Medicaid services. [Respir Care 2016;61(8):1023-1032. @ 2016 Daedalus Enterprises]
\end{abstract}

\section{Introduction}

Sleep medicine frequently operates under the policies of insurance carriers that view positive airway pressure (PAP)

\footnotetext{
Dr Krakow, Mr Ulibarri, and Ms McIver are affiliated with the Sleep and Human Health Institute, Albuquerque, New Mexico. Dr Krakow, Mr Ulibarri, Ms Foley-Shea, Ms Tidler, and Ms McIver are affiliated with Maimonides Sleep Arts and Sciences, Ltd, Albuquerque, New Mexico. Dr Krakow is also affiliated with the Los Alamos Medical Center, Los Alamos, New Mexico.
}

therapy as an all-or-nothing therapeutic device for sleep apnea patients. ${ }^{1}$ In the United States ${ }^{2}$ and internationally, ${ }^{3-5}$ numerous industrialized countries use compliance metrics based

\footnotetext{
Dr Krakow has disclosed a relationship with ResMed and has conducted continuing medical education/continuing education units programs for sleep disorders for medical and mental health providers and vendors, such as Respironics, ResMed, the Army Medical Department Center and School, and regional sleep center conferences. The other authors have disclosed no conflicts of interest.
} 


\section{AdHerence in Subjects With OSA Receiving CPAP}

either on the Medicare policy of $4 \mathrm{~h} /$ night for $70 \%$ of nights/week or variations thereof., 2,6-8 Although such policies manifest great influence over clinical care, they are based only on descriptive studies (Level V: lowest-rated evidence on Sackett's criteria ${ }^{9}$ ) in lieu of rigorous scientific investigations. In this context, imagine a diabetic patient using only $50 \%$ of prescribed drugs and then being informed by her doctor: Since you're not using $70 \%$ or greater, insurance coverage ceases for your oral anti-hyperglycemics. Worse, imagine the physician asserting: Despite improvement in fasting blood sugars and well-being with the lower dosage, you must return your medication or pay for it out of pocket.

This incongruous scenario is accepted practice in the field of sleep medicine in the United States ${ }^{1}$ and various parts of the world, ${ }^{3-5}$ presumably because insurance carriers perceive unacceptable levels of waste in patients not using their PAP devices. ${ }^{10,11}$ Universally reported noncompliance issues in sleep apnea patients may be compounded by adversarial relationships between sleep centers and durable medical equipment companies, ${ }^{12}$ resulting in a lack of timely interventions to resolve early adverse effects caused by the device..$^{8,13-17}$

This commonly used adherence paradigm appears to have been adopted prematurely without having been subjected to in-depth investigations to discover a beneficial patientcentered metric. From a clinical standpoint, the current metric may promote lesser quality of care and loss of potential healthcare cost savings due to precipitous termination of PAP coverage. ${ }^{10}$ In contrast, one respiratory medical association has declared that patients should be considered compliant

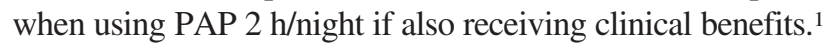
Also, research to date on subjects with subthreshold compliant PAP use has consistently shown improvements in subjective or objective sleepiness, despite the lower number of objective hours recorded (Table 1).18-22

Two factors might yield a validated approach to adherence: (1) measurement of actual hours of PAP use as a continuous variable, reflecting a potential dose-response relationship and (2) impact of use hours on health-care outcomes and related cost savings. Studies already document improved outcomes ${ }^{23,24}$ and health-care cost reductions ${ }^{25,26}$ associated with regular use of PAP therapy, yet, to our knowledge, the putative dose-response effect of PAP therapy may not be considered by those outside the

Supplementary material related to this paper is available at http:// www.rcjournal.com.

Correspondence: Barry Krakow MD, Sleep and Human Health Institute, 6739 Academy NE, Suite 380, Albuquerque, NM 87109. E-mail: bkrakow@sleeptreatment.com.

DOI: $10.4187 /$ respcare 04538

\section{QUICK LOOK}

\section{Current knowledge}

The concept of therapeutic dose response is well-established. However, positive airway pressure (PAP) treatment for sleep apnea is constrained by policies that apply a "use it or lose it" principle derived from an arbitrary compliance definition involving a set minimum hours of PAP use. However, subthreshold compliant patients and other partial users who fall below the minimum hours also report benefits.

\section{What this paper contributes to our knowledge}

This study shows that subcompliant PAP patients gain improvements in subjective insomnia, sleepiness, and nocturia symptoms, which lend clear support for a PAP dose-response relationship. These findings also mirror the clinical concept of transitional adaptation in which patients use a drug therapy and incrementally increase the dosage over time while gradually gaining more benefits. In sleep medicine, arbitrary PAP compliance policies may serve insurance carriers, but they appear counterproductive for patients in their early adaptation period. Focusing on patients' use of PAP therapy may ultimately improve their adherence.

medical profession, although anecdotally, a doseresponse relationship aligns with a gradual adaptation process observed in some sleep apnea patients. ${ }^{17}$ The pivotal work of Stepnowsky and Moore ${ }^{27}$ on the doseresponse relationship examined trials with PAP and sham or placebo devices, noting clear benefits from subtherapeutic levels of pressurized air. They offered 2 essential insights. First, "[u]nderstanding the dose-response relationship between CPAP and important outcomes will put compliance intervention studies into perspective." Second, "... the fact that we do not understand the scope and consequences of CPAP use and 'dose' presents itself as a major research mission."

Additional studies ${ }^{28-35}$ as well as the salient commentary of Brown ${ }^{36}$ on the topic of subcompliant PAP use strongly suggest the clinical utility of a dose-response between hours of use and improvement in outcomes (sleep apnea hypopnea syndrome symptoms, ${ }^{30,32}$ cognitive function, ${ }^{33,34}$ and mortality ${ }^{28}$ ) among subthreshold compliant patients using PAP on a regular basis but for fewer hours. These works challenge policy directives and suggest that quality of care would be enhanced by thinking in terms of hours of use or time on mask instead of relying solely on compliance or adherence constructs.

To replicate past research as well as analyze possible doseresponse relationships, we retrospectively compared sleepi- 


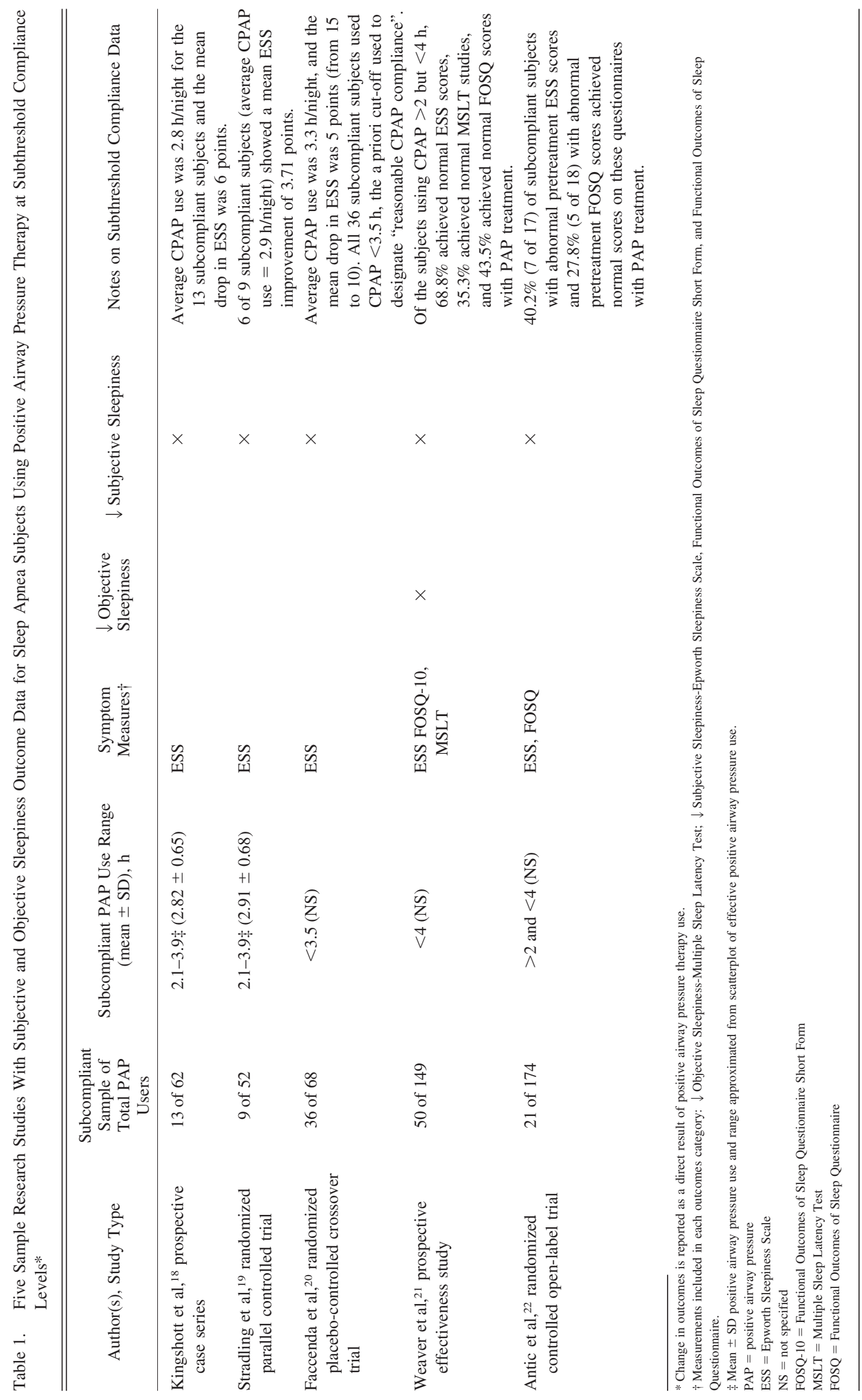




\section{AdHerence in Subjects With OSA Receiving CPAP}

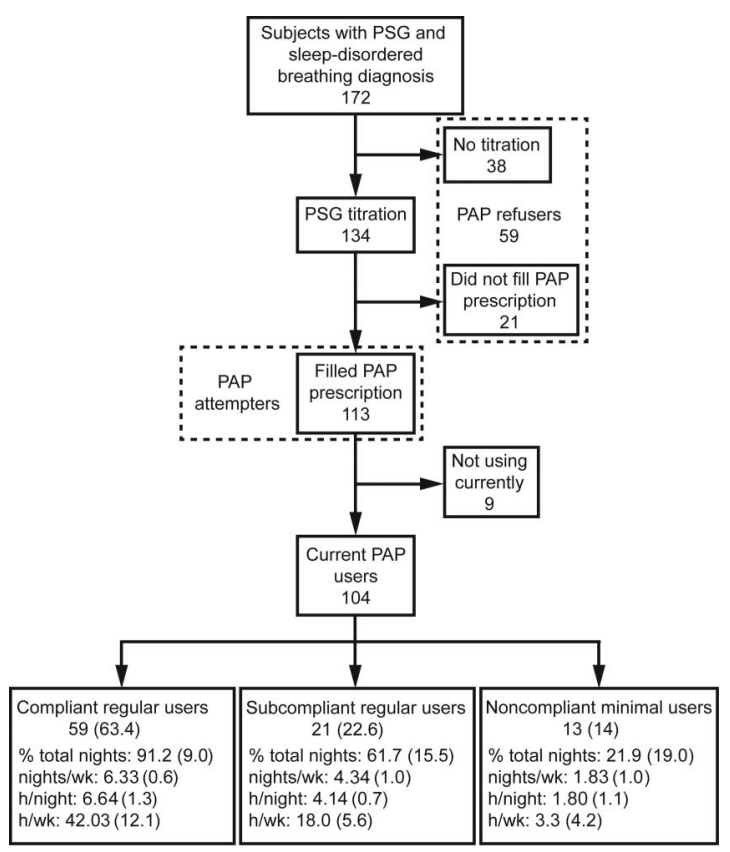

Fig. 1. Flow chart. PSG = polysomnography, $\mathrm{PAP}=$ positive airway pressure. Note only 93 of the 104 current PAP users were categorized into the 3 subject groups.

ness outcomes in compliant and subthreshold compliant subjects, and we evaluated changes in 2 outcomes, insomnia and nocturia, not previously researched in subthreshold compliant sleep apnea cases. ${ }^{37-43} \mathrm{~A}$ consecutive series of subjects (Fig. 1) who presented to our community-based sleep medical center (Maimonides Sleep Arts \& Sciences, Ltd.) were divided into compliant regular users, subthreshold regular users, and minimal users. We hypothesized that despite significant differences in total weekly hours used by each group, compliant and subthreshold users would manifest clinically meaningful improvements (by effect sizes) in sleepiness, insomnia, and nocturia episodes compared with minimal users. Also, we further hypothesized that a significant dose-response relationship would manifest between PAP use hours and changes in outcomes.

\section{Methods}

\section{Study Criteria and Consent}

This retrospective chart review assessed adult, PAPnaive subjects presenting from June through December, 2013, who (1) were objectively diagnosed with obstructive sleep apnea (OSA) or upper airway resistance syndrome (UARS); (2) completed a titration polysomnogram; and (3) filled a prescription for PAP. Per standard protocol, all subjects provided verbal and written consent for anonymous use of medical information for research and educational purposes in the context of chart and data reviews.
All subjects' intakes were reviewed by the medical director (BK) before diagnostic polysomnography. This project was approved by the Los Alamos Medical Center institutional review board.

\section{Chart Review, Polysomnography, Breathing Event Metrics}

Maimonides Sleep Arts \& Sciences patients complete online intakes assessing sleep symptoms based on the nosology for sleep disorders in the International Classification of Sleep Disorders. ${ }^{44}$ The intake also includes 2 validated surveys, the Insomnia Severity Index ${ }^{45}$ and Epworth Sleepiness Scale, ${ }^{46}$ and a query on frequency of nocturia episodes per night. Measures are repeated at follow-up, several months after initiation of PAP therapy. Polysomnography was conducted and scored using standard American Academy of Sleep Medicine guidelines ${ }^{47}$ as well as standard rules for calculating the apnea-hypopnea index (AHI) and respiratory disturbance index (RDI). ${ }^{47,48}$ Similar to models recently described in ReSPIRATORY CARE, 49,50 we also engage patients to view select polysomnographic tracings of their sleep studies to enhance their education (the supplementary materials at http://www.rcjournal.com describe additional scoring details and our titration practice model, including use of ABPAP and ASV PAP devices to increase patient comfort and compliance).

\section{PAP Use and Adherence}

Among the final sample, current users were distinguished from non-users based on the presence of these recent encounters: (1) prescription renewal for PAP supplies ${ }^{51}$; (2) clinic appointment regarding continued PAP use; (3) retitration and confirmation of use; or (4) contact with office staff, discussing continued PAP use. Among subjects with objective data downloads, nightly hours were calculated as average hours of PAP use for all nights and average hours of PAP use for only the nights used. Three compliance groups were created from the objective data downloads: (1) compliant regular users, who met Centers of Medicare and Medicaid Services (CMS) compliance guidelines (mean PAP use $\geq 4 \mathrm{~h} /$ night on $70 \%$ of nights); (2) subcompliant regular users, who were PAP users not meeting CMS guidelines but who averaged regular, nightly use $>3 \mathrm{~h} /$ night; and (3) noncompliant minimal users, who had minimal PAP use (averaging $<2 \mathrm{~h} /$ night).

\section{Sample and Data Analyses}

Figure 1 describes the flow of PAP-naive subjects seeking care during the 6-month study period. One hundred seventy-two subjects completed their intake and were objectively diagnosed with either OSA $(n=154$; mean \pm SD 


\section{AdHerence in Subjects With OSA Receiving CPAP}

AHI $29.52 \pm 26.53$ and RDI $62.58 \pm 26.47$ ) or UARS $(n=18$; mean \pm SD AHI $2.17 \pm 1.34$ and RDI $50.46 \pm 20.42$ ). Of the 172 subjects, 134 completed a fullnight titration, and 21 failed to fill their PAP prescription, leaving 113 subjects who moved forward to initiate treatment with a PAP device at home.

Analysis of variance compared continuous variables. The chi-square test analyzed dichotomous variables. For subjects with objective data downloads, 2-way repeatedmeasures analysis of variance for Insomnia Severity Index, Epworth Sleepiness Scale, and nocturia episodes per night compared values pre- and post-PAP use; Cohen's d effect size $(0.2=$ small, $0.5=$ medium, $0.8=$ large $)$ calculated the standardized difference between 2 means within each subsample for the 3 compliance groups. Objective data downloads also provided AHI and mask leak values at follow-up for between-group comparisons. The Pearson product moment correlation coefficient analyzed relationships for changes in Insomnia Severity Index, Epworth Sleepiness Scale, and nocturia episodes per night by hours of PAP use/week. A $P$ value of .05 was statistically significant. Data were analyzed with IBM SPSS Statistics version 11.0 for Windows (IBM Corporation, Armonk, New York).

\section{Results}

\section{Sample Characteristics and Use Groups}

Our final sample of 113 subjects was predominantly middle age $(54.4 \pm 12.4 \mathrm{y})$, minimally obese (body mass index $\left.=31.23 \pm 6.81 \mathrm{~kg} / \mathrm{m}^{2}\right)$, white $(73.5 \%)$ and Hispanic $(21.2 \%)$, married $(73.5 \%)$, male $(57.5 \%)$, and with a bachelor's degree or higher $(57.5 \%)$. Psychiatric history included depression $(38.9 \%, n=44)$, anxiety disorder $(23.9 \%, n=27)$, post-traumatic stress disorder $(9.7 \%$, $n=11)$, panic attacks $(11.5 \%, n=13)$, trauma exposure $(28.3 \%, n=32)$, claustrophobia $(23.9 \%, n=27)$, and bipolar illness $(1.8 \%, n=2)$. Only $30.1 \%(n=34)$ denied psychiatric history or complaints. For main outcomes, mean nocturia episodes per night were $1.55 \pm 1.17$, and Insomnia Severity Index and Epworth Sleepiness Scale scores were $13.69 \pm 5.42$ and $9.69 \pm 4.72$, respectively, the latter measure reflecting a subthreshold level of clinical sleepiness in our subject population who presented with mental health symptoms. Based on our definitions, 104 of 113 subjects $(92 \%)$ were using PAP at follow-up (mean \pm SD period post-initiation of $\mathrm{PAP}=7.23 \pm 2.20$ months), and 9 subjects ( $8 \%$ ) were not (Fig. 1).

\section{Compliance Category and Subgroups by Objective Data Downloads}

Among the 104 PAP users, 93 current objective data downloads categorized subjects into 3 subgroups described in Methods (compliant regular users, $n=59$; subthreshold regular users, $n=21$; and noncompliant minimal users, $n=13$ ). There were no significant differences in sociodemographic or intake data among the groups.

In Figure 1, the percentage of nights used and average PAP hours/week increased from noncompliant minimal users to subcompliant regular users, and the largest values were seen in compliant regular users. Both the compliant regular user and subcompliant regular user groups averaged $>4 \mathrm{~h}$ of PAP use/nights used, but subcompliant regular users applied PAP on average just over 4 nights/week (adherence standards require 5 nights/week), whereas compliant regular users averaged 6+ nights of PAP use/week. In contrast, noncompliant minimal users averaged $1.80 \mathrm{~h}$ of PAP use/nights used and between 1 and 2 nights/week, both metrics below adherence standards.

Of clinical import, 8 of 21 subjects (38\%) in the subcompliant regular user group averaged $>4 \mathrm{~h} /$ night on nights used for $>20 \mathrm{~h} /$ week but used PAP on $<70 \%$ of nights. Thus, nearly $40 \%$ of subcompliant regular user subjects would meet standards if total hours/week $(\sim 20 \mathrm{~h})$ was the policy instead of the arbitrary rule of $70 \%$ of nights with $\geq 4 \mathrm{~h} /$ night use (ie, 4.9 nights $\times 4 \mathrm{~h}=19.6 \mathrm{~h}$ ). Two subjects highlight this distinction: One from the subcompliant regular user group was noncompliant despite nightly PAP use (100\%) for $3.62 \mathrm{~h} / \mathrm{hight}$ on average totaling $25.3 \mathrm{~h} /$ week in contrast to the other case, a compliant subject who used PAP for $4.57 \mathrm{~h}$ on 6 nights/week for a total of $27.4 \mathrm{~h}$. In 2 more subcompliant regular user cases, one subject used 5.08 $\mathrm{h} /$ night for $68.29 \%$ of nights equaling $24.28 \mathrm{~h} /$ week, and another subject used $4.42 \mathrm{~h} /$ night for $68.42 \%$ of nights equaling $21.17 \mathrm{~h} /$ week; thus, both cases were noncompliant. Figure 2 focuses on these distinctions by graphically highlighting a transitional zone comprising 26 subjects ( 8 compliant regular users [squares] and 18 subcompliant regular users [diamonds]) who display borderline values that reach just above or below compliance.

\section{Changes in Outcome Measures By Subgroup Analysis}

For 93 subjects with subjective and objective data, outcomes (insomnia, sleepiness, nocturia episodes) revealed no significant differences among groups at baseline: Insomnia Severity Index ranged from mild to moderate severity; Epworth Sleepiness Scale ranged from subclinical to mild daytime sleepiness; and nocturia episodes per night were in the 1-2-episode/night range. At follow-up (Fig. 3), compliant regular users showed the largest changes (mean intake to outcome) in all scales, with moderate to large effects for insomnia $(13.53 \pm 0.66$ to $8.88 \pm 0.69 ; \mathrm{d}=0.94)$, sleepiness $(9.09 \pm 0.52$ to $6.77 \pm 0.58 ; \mathrm{d}=0.58)$, and nocturia $(1.56 \pm 0.14$ to $1.06 \pm 0.16 ; \mathrm{d}=0.56)$, all significant $(P<.05)$. Subcompliant regular users showed a 


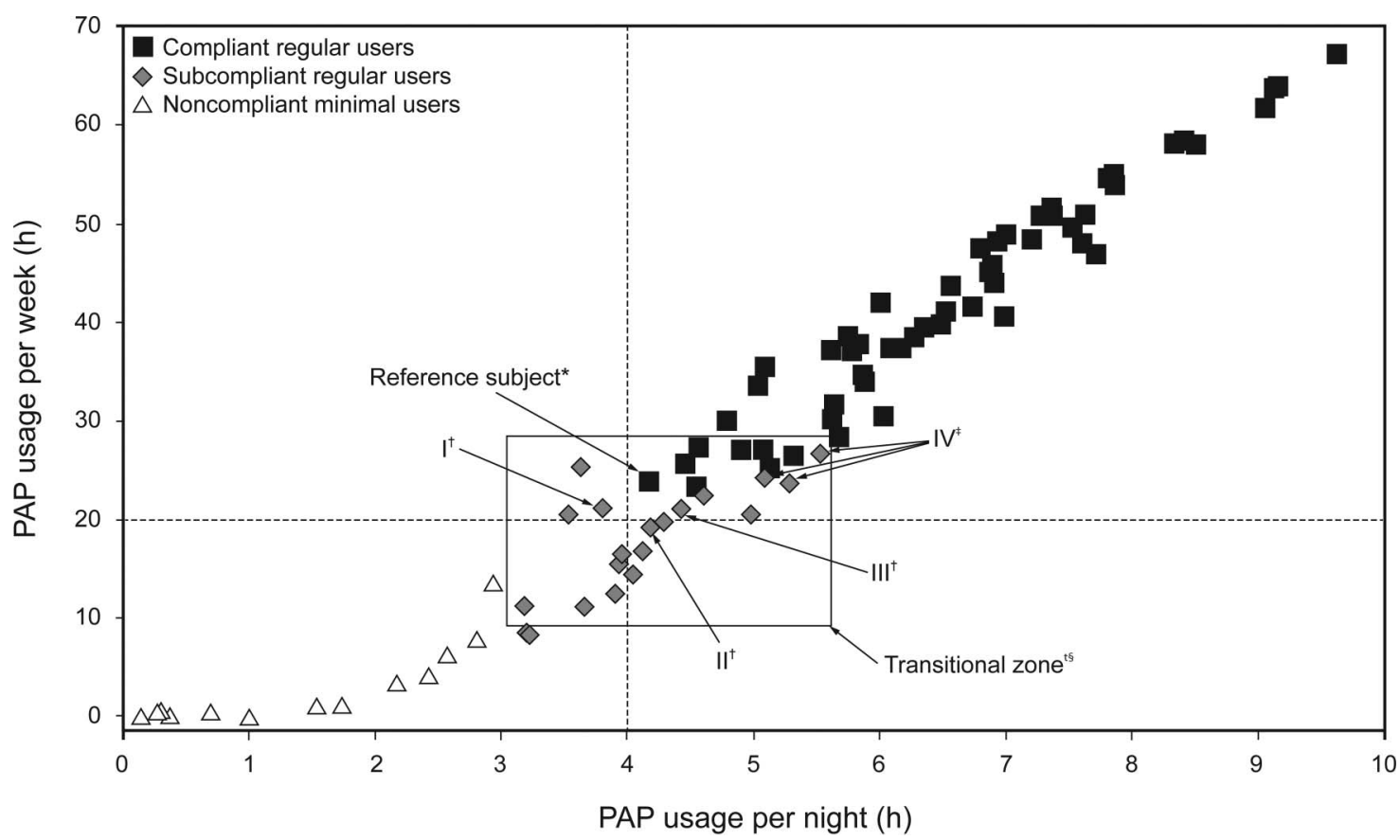

Fig. 2. Scatter plot of weekly PAP hours used [nightly PAP average (h/night) X \% nights used (nights/week)] for compliant regular users, subcompliant regular users, and noncompliant minimal users. The vertical dashed line signifies a compliance standard of $4 \mathrm{~h} / \mathrm{night}$, whereas the horizontal dashed line represents $19.6 \mathrm{~h}$ (70\% nights/week X 4h/night), which is the minimum standard for CMS coverage provided patient meets additional criteria: $\geq 5$ nights/week, $\geq 4$ hours/night used. However, values above this line do not guarantee compliance as many subjects used $<5$ days/week or $<4 \mathrm{hrs} /$ night. The transitional zone contains subjects with borderline use values just above (compliant regular users; $n=8$ ) or just below (subcompliant regular users; $n=18$ ) compliance guidelines. *The Reference subject is the compliant regular user with the least amount of nightly use among all compliant subjects. †Subjects I, II, and III are subcompliant regular users with weekly use values near to Reference subject, yet noncompliant for various reasons: Subject I, $21.1 \mathrm{~h} /$ week, $79.4 \%$ nights, but $3.8 \mathrm{~h} / \mathrm{hight}$; Subject II, 4.2 h/night, but 19.2 h/week, and 65.9\% nights; Subject III, 21.2 h/week, 4.4 h/night, but 68.4\% nights; ¥Subjects IV, subcompliant regular users with PAP hours/week more than compliant Reference subject. Our sample used two different PAP modes [ASV ( $n=69)$ and $\operatorname{ABPAP}(n=24)]$ at time of follow-up.

significant, large effect for insomnia $(13.83 \pm 1.29$ to $9.17 \pm 1.38 ; \mathrm{d}=0.76$ ), a nonsignificant, small to medium effect for sleepiness $(9.78 \pm 1.09$ to $7.72 \pm 1.27 ; \mathrm{d}=0.38)$, and a small effect for nocturia $(1.58 \pm 0.31$ to $1.33 \pm 0.18$; $\mathrm{d}=0.22)$. Last, noncompliant minimal users showed a small to medium effect $(12.08 \pm 1.39$ to $10.25 \pm 1.47$; $\mathrm{d}=0.36)$ for insomnia, a small effect for sleepiness (8.83 \pm 1.81 to $7.67 \pm 1.55 ; \mathrm{d}=0.19)$, and no change for nocturia $(0.88 \pm 0.32$ to $0.88 \pm 0.15 ; \mathrm{d}=0.00)$.

For all 93 subjects with objective data downloads, we determined bivariate correlation coefficients for weekly hours of use by change in score for each measure of insomnia, sleepiness, and nocturia (Fig. 4). For insomnia $(\mathrm{r}=.20)$ and nocturia $(\mathrm{r}=.25)$, a trend or significance, respectively, was observed in a direction suggesting a doseresponse relationship associated with PAP use.

\section{Supplemental Analyses}

As further corroboration of PAP efficacy among all 93 subjects with objective data downloads, we collected re- sidual AHI and objective leak data. All 3 groups averaged AHI $<2$ (range $1.19 \pm 2.03$ to $1.80 \pm 2.66$; interquartile range $=1.30 ;$ median $=0.80)$ with no significant differences. For leak (L/min), the groups ranged from $6.73 \pm 7.76$ to $10.86 \pm 12.24$ (interquartile range $=12.45$; median $=3.60$ ) with no significant differences.

For PAP refusers described in Figure 1, 59 subjects did not attempt PAP therapy, including 38 who chose not to complete a titration and 21 who completed a titration but chose not to fill a prescription. Reasons offered for no follow-through are listed in eTable 1 (see supplementary material). There were no significant differences between 59 refusers and 113 attempters or between 59 refusers and 104 current users.

\section{Discussion}

In this retrospective review of 113 subjects who completed a sleep apnea evaluation and filled a prescription to initiate PAP therapy, 104 were using a device at followup. Data downloads on 93 users demonstrated 59 compli- 

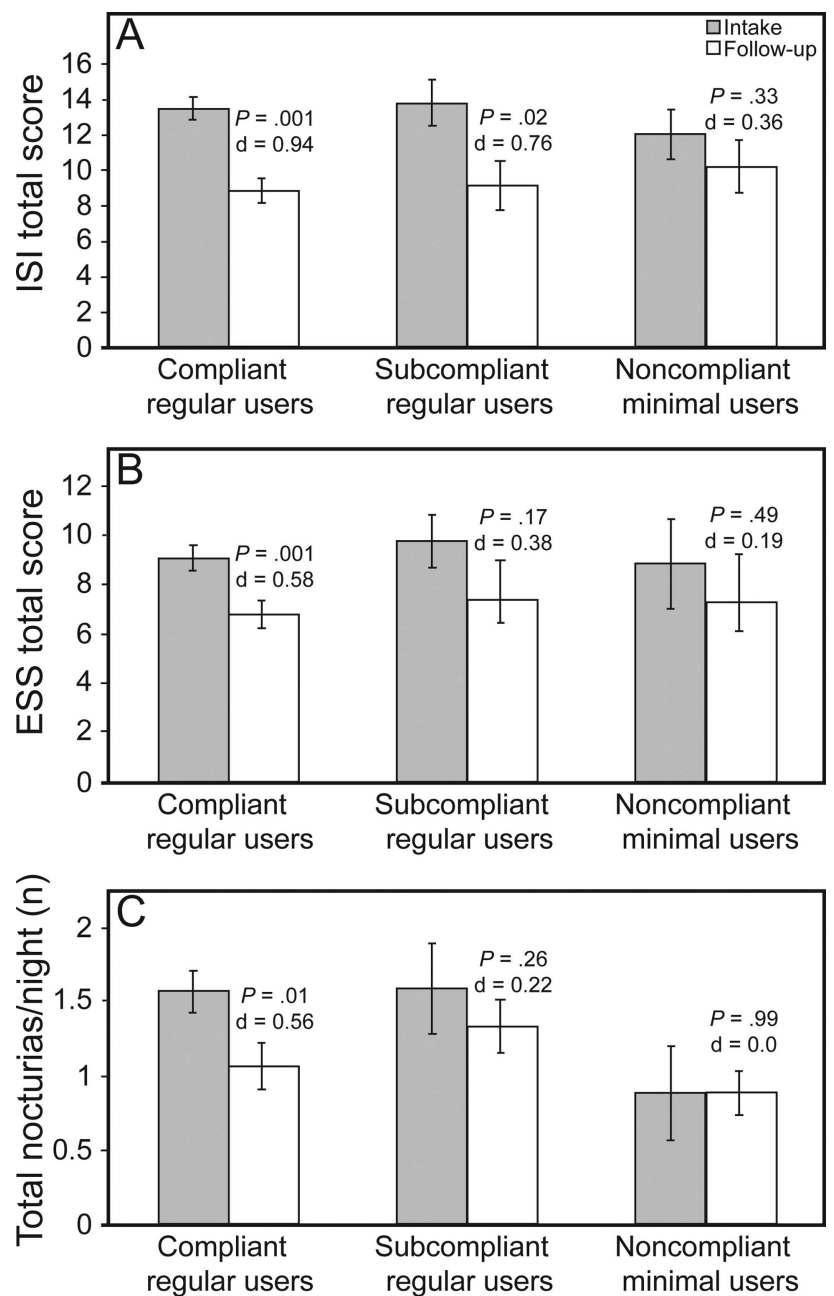

Fig. 3. Comparison of mean $\pm S D$ intake versus follow-up values A: Insomnia Severity Index total score, B: Epworth Sleepiness Scale total score, and C: total nocturia episodes per night for compliant regular users $(n=59)$, subcompliant regular users $(n=$ 21 ), and noncompliant minimal users $(n=13)$. Scores for Insomnia Severity Index, Epworth Sleepiness Scale, and nocturia episodes expressed as mean $\pm S D$ and analyzed with repeated-measures analysis of variance. $P$ values are for the change in score from intake to outcome; $d=$ Cohen's $d$ effect size $(0.2=$ small, $0.5=$ medium, 0.8 = large) calculated the standardized difference between 2 means within each subsample for the 3 compliance groups.

ant, 21 subthreshold compliant, and 13 noncompliant minimal users. Of clinical import, 21 subcompliant, regular users of PAP therapy averaged improvements (noted in small to large effect sizes) in primary variables of selfreported insomnia, sleepiness, and nocturia, albeit sample sizes may have affected significance testing. Paradoxically, these cases could have had their therapy withdrawn had they been subjected to insurance compliance standards despite the benefit they were clearly receiving from relatively lower levels of use. This disadvantage directed at sleep apnea patients is well formulated in Brown's commentary: "A substantial number of [policies] apply to costly devices ...
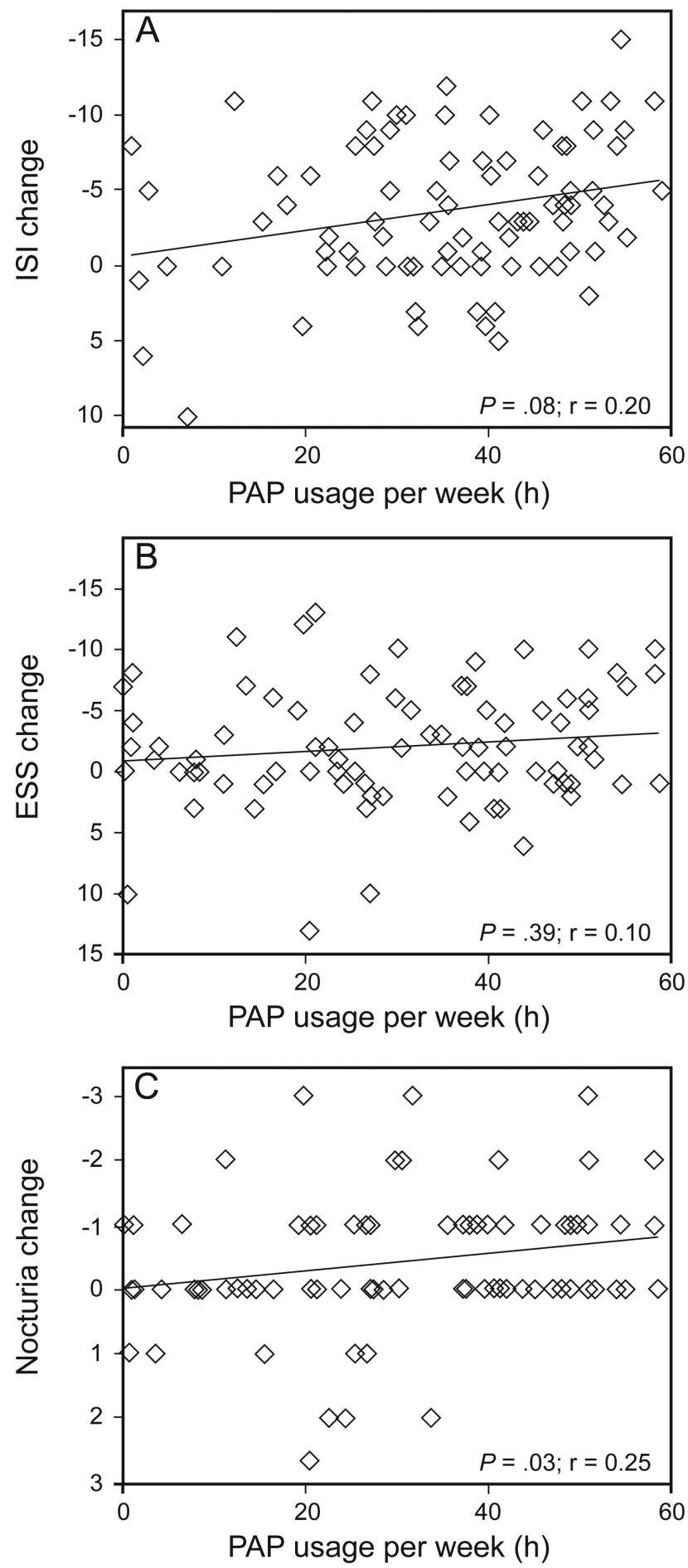

Fig. 4. Bivariate correlation between PAP use (hours/week) ${ }^{\star}$ and change in value from intake to outcome for (A) Insomnia Severity Index (ISI), (B) Epworth Sleepiness Scale (ESS), and (C) total nocturia episodes. PAP hours used/week obtained from the most recent objective data download.

equipped for objective measurement of usage, including oxygen concentrators, mechanical in-ex-sufflation devices, power wheelchairs, and pneumatic compression apparatus; 


\section{AdHerence in Subjects With OSA Receiving CPAP}

however, only PAP and respiratory assist devices are singled out in this manner [ie., measuring hours of use] ..."36

Our work extends findings from several researchers who demonstrated similar results among patients whose hours of use fall below adherence standards but yield improvement in outcomes, ${ }^{18-22,28-34}$ albeit our findings on changes in sleepiness were not as robust, probably due to low baseline Epworth scores in our mental health subjects. To our knowledge, this study is the first to demonstrate improved outcomes for insomnia and possibly nocturia (based on effect size) in regular users of PAP therapy who did not meet compliance standards. Consistent, albeit small, correlation coefficients were observed for the relationships between hours of use (time on mask) and insomnia severity (trend) and nocturia episodes (significance). Taken together, the current study supports a theory of a dose-response for PAP therapy, suggesting the importance of viewing hours of use as a continuous variable; this perspective matches conventional wisdom as applied to many other therapeutic interventions, notably medications.

The findings in this study may further our capacity to distinguish between the terms "use of" as opposed to "adherence with" PAP devices. Clinically, we presume that most sleep medicine specialists encounter minimal use patients who attain subthreshold status as well as those initially subthreshold who later attain full adherence. This point is highlighted in the Figure 2 transitional zone box wherein the "Reference Subject" (least amount of average nightly use among all compliant subjects) demonstrates only slightly higher weekly use ( $23.77 \mathrm{~h})$ compared with 3 very nearly adherent, subthreshold subjects: $21.13 \mathrm{~h}$ (I), $19.23 \mathrm{~h}$ (II), and $19.80 \mathrm{~h}$ (III). In related examples, 3 other subthreshold subjects in the transitional zone (IV) actually used PAP for the same or more total hours/week than the reference subject, yet none were adherent. As can be calculated, mere minutes per night separated these subjects from attaining insurance coverage or requiring self-payment or, worse, therapy withdrawal despite improved outcomes. If, on the other hand, a dose-response relationship were considered, taking into account the benefits that these subjects achieved in daytime sleepiness, insomnia, and nocturia with a lower dosage of therapy, then patient care could be enhanced.

Summing up, we would express concern for any system that discourages recognition of these transitional scenarios, especially since the original compliance construct for PAP use was based on low-evidence studies. ${ }^{1,2}$ A scientifically determined dose-response metric devised by experts from the field of sleep medicine appears to be a more promising approach to the short- and long-term management of OSA/UARS patients. More research in this area could bolster sleep medicine specialists' efforts to negotiate on behalf of struggling patients who are receiving benefits although not yet adherent. ${ }^{1,36}$ Failing to attend to this dose-response relationship may adversely affect patient care, especially among those who require longer intervals to adapt to PAP therapy, irrespective of the financial burdens imposed by insurance rules. Additional research on the dose-response (the conventional approach to medical care in general) of PAP therapy might also raise the standard of care in sleep medicine by supporting higher levels of evidence in clinical practice to treat patients in lieu of arbitrary insurance rules.

\section{Limitations}

This work is limited by a lack of a larger and more diverse sample, which would provide more generalizable findings and yield more power for statistical testing of subthreshold compliant patients. Then again, a larger sample might not validate these results, and therefore our findings should be viewed with caution. A prospective, longitudinal research design would yield a great deal more insight into the waxing and waning patterns regarding the time on mask that occurs among PAP therapy patients for whom a wide range of hours of use is most likely the norm. ${ }^{17}$ Whereas dose-response changes in outcomes were measured in this study, cost-savings calculations were not available, and this latter factor could prove an essential parameter through which to influence policies currently promoting the construct of compliance instead of use. Another limitation is the preponderance of subjects suffering from comorbid psychiatric conditions, which may also reduce generalizability, albeit a number of recent works suggest that a substantial proportion of treatment-seeking OSA subjects report a history of mental health conditions or use of psychotropic medications. ${ }^{52-57}$ Last, among the subgroups who did not pursue PAP (no titration or prescription), anecdotal insights suggest that 19 patients specifically refused this treatment very early in the process, which may relate to the work of Brostrom et $\mathrm{al}^{58}$ on PAP rejection by subjects with Type D personality (eTable 1).

In conclusion, although our retrospective design demands a cautious interpretation of findings, our data and the data of prior researchers suggest a putative dose-response effect from PAP therapy. The time line of PAP use for any OSA/UARS patient may emerge in ways similar to the medical practice of attempting to increase the dosage of a drug while both early benefits and adverse effects occur at initial doses, a pattern common to antidepressant medications. ${ }^{59}$ Since many patients demonstrate a capacity to endure a therapeutic process more gradually, it seems perplexing that so much emphasis has been placed on an arbitrarily devised adherence construct. Sleep medicine professionals are focused on encouraging greater PAP use and no doubt incorporate naturalistic approaches aligned with patients' learning curves, but adherence rules may interfere with this general practice model of care for select 


\section{AdHerence in Subjects With OSA Receiving CPAP}

patients. In the real world, given that PAP benefits accrue during a variable time line ${ }^{60}$ like so many medications, the concept of use may provide a more pragmatic clinical tool in patient management, and speculatively, a use framework may ultimately lead to greater compliance.

\section{ACKNOWLEDGMENTS}

We thank the Los Alamos Medical Center and the Los Alamos Medical Center Sleep Laboratory for administrative and clinical assistance in the completion of this research project.

\section{REFERENCES}

1. Schwab RJ, Badr SM, Epstein LJ, Gay PC, Gozal D, Kohler M, et al. An official American Thoracic Society statement: continuous positive airway pressure adherence tracking systems: the optimal monitoring strategies and outcome measures in adults. Am J Respir Crit Care Med 2013;188(5):613-620.

2. Kribbs NB, Pack AI, Kline LR, Smith PL, Schwartz AR, Schubert $\mathrm{NM}$, et al. Objective measurement of patterns of nasal CPAP use by patients with obstructive sleep apnea. Am Rev Respir Dis 1993; 147(4):887-895

3. Ayas N, Skomro R, Blackman A, Curren K, Fitzpatrick M, Fleetham $\mathrm{J}$, et al. Obstructive sleep apnea and driving: a Canadian Thoracic Society and Canadian Sleep Society position paper. Can Respir J 2014;21(2):114-123.

4. Kim JH, Kwon MS, Song HM, Lee BJ, Jang YJ, Chung YS. Compliance with positive airway pressure treatment for obstructive sleep apnea. Clin Exp Otorhinolaryngol 2009;2(2):90-96

5. European Sleep and Diagnostics Reimbursement Overview. ResMed, 2015. Correspondence from ResMed.

6. Reeves-Hoche MK, Meck R, Zwillich CW. Nasal CPAP: an objective evaluation of patient compliance. Am J Respir Crit Care Med 1994;149(1):149-154

7. Salepci B, Caglayan B, Kiral N, Parmaksiz ET, Comert SS, Sarac G, et al. CPAP adherence of patients with obstructive sleep apnea. Respir Care 2013;58(9):1467-1473.

8. Weaver TE, Grunstein RR. Adherence to continuous positive airway pressure therapy: the challenge to effective treatment. Proc Am Thorac Soc 2008;5(2):173-178

9. Sackett DL, Straus SE, Richardson WS, Rosenberg W, Haynes RB. Evidence-based medicine: how to practice and teach EBM. 2nd edition. Edinburgh, Scotland: Churchhill Livingstone; 2000:173-177.

10. Billings ME, Kapur VK. Medicare long-term CPAP coverage policy: a cost-utility analysis. J Clin Sleep Med 2013;9(10):1023-1029.

11. Wolkove N, Baltzan M, Kamel H, Dabrusin R, Palayew M. Longterm compliance with continuous positive airway pressure in patients with obstructive sleep apnea. Can Respir J 2008;15(7):365-369.

12. Anderson L. In search of compliance. http://www.homecaremag .com/hme-products/search-compliance Cahaba Media Group, Home Care 2012.

13. Borel JC, Tamisier R, Dias-Domingos S, Sapene M, Martin F, Stach $\mathrm{B}$, et al. Type of mask may impact on continuous positive airway pressure adherence in apneic patients. PLoS One 2013;8(5):e64382.

14. Weaver TE, Kribbs NB, Pack AI, Kline LR, Chugh DK, Maislin G, et al. Night-to-night variability in CPAP use over the first three months of treatment. Sleep 1997;20(4):278-283.

15. Wild MR, Engleman HM, Douglas NJ, Espie CA. Can psychological factors help us to determine adherence to CPAP? A prospective study. Eur Respir J 2004;24(3):461-465

16. Broström A, Nilsen P, Johansson P, Ulander M, Strömberg A, Svanborg E, Fridlund B. Putative facilitators and barriers for adherence to
CPAP treatment in patients with obstructive sleep apnea syndrome a qualitative content analysis. Sleep Med 2010;11(2):126-130.

17. Bollig SM. Encouraging CPAP adherence: it is everyone's job. Respir Care 2010;55(9):1230-1239.

18. Kingshott RN, Vennelle M, Hoy CJ, Engleman HM, Deary IJ, Douglas NJ. Predictors of improvements in daytime function outcomes with CPAP therapy. Am J Respir Crit Care Med 2000;161(3 Pt 1): 866-871.

19. Stradling JR, Davies RJ. Is more NCPAP better? Sleep 2000;23(Suppl 4):S150-S153.

20. Faccenda JF, Mackay TW, Boon NA, Douglas NJ. Randomized placebo-controlled trial of continuous positive airway pressure on blood pressure in the sleep apnea-hypopnea syndrome. Am J Respir Crit Care Med 2001;163(2):344-348.

21. Weaver TE, Maislin G, Dinges DF, Bloxham T, George CF, Greenberg $\mathrm{H}$, et al. Relationship between hours of CPAP use and achieving normal levels of sleepiness and daily functioning. Sleep 2007;30(6): 711-719.

22. Antic NA, Catcheside P, Buchan C, Hensley M, Naughton MT, Rowland S, et al. The effect of CPAP in normalizing daytime sleepiness, quality of life, and neurocognitive function in patients with moderate to severe OSA. Sleep 2011;34(1):111-119.

23. Engleman HM, Martin SE, Deary IJ, Douglas NJ. Effect of continuous positive airway pressure treatment on daytime function in sleep apnoea/hypopnoea syndrome. Lancet 1994;343(8897):572-575.

24. Martinez-Garcia MA, Soler-Cataluna JJ, Ejarque-Martinez L, Soriano Y, Roman-Sanchez $\mathrm{P}$, Illa FB, et al. Continuous positive airway pressure treatment reduces mortality in patients with ischemic stroke and obstructive sleep apnea: a 5-year follow-up study. Am J Respir Crit Care Med 2009;180(1):36-41.

25. McDaid C, Griffin S, Weatherly H, Duree K, van der BM, van Hout $\mathrm{S}$, et al. Continuous positive airway pressure devices for the treatment of obstructive sleep apnoea-hypopnoea syndrome: a systematic review and economic analysis. Health Technol Assess 2009;13(4): iii-iv, xi-xiv, 1-119.

26. Peker Y, Hedner J, Johansson A, Bende M. Reduced hospitalization with cardiovascular and pulmonary disease in obstructive sleep apnea patients on nasal CPAP treatment. Sleep 1997;20(8):645-653.

27. Stepnowsky CJ Jr, Moore PJ. Nasal CPAP treatment for obstructive sleep apnea: developing a new perspective on dosing strategies and compliance. J Psychosom Res 2003;54(6):599-605.

28. Campos-Rodriguez F, Peña-Griñan N, Reyes-Nuñez N, De la CruzMoron, I, Perez-Ronchel J, De la Vega-Gallardo F, Fernandez-Palacin A. Mortality in obstructive sleep apnea-hypopnea patients treated with positive airway pressure. Chest 2005;128(2):624-633.

29. Engleman HM, Cheshire KE, Deary IJ, Douglas NJ. Daytime sleepiness, cognitive performance and mood after continuous positive airway pressure for the sleep apnoea/hypopnoea syndrome. Thorax 1993;48(9):911-914.

30. Engleman HM, Kingshott RN, Wraith PK, Mackay TW, Deary IJ, Douglas NJ. Randomized placebo-controlled crossover trial of continuous positive airway pressure for mild sleep apnea/hypopnea syndrome. Am J Respir Crit Care Med 1999;159(2):461-467.

31. Jenkinson C, Davies RJ, Mullins R, Stradling JR. Comparison of therapeutic and subtherapeutic nasal continuous positive airway pressure for obstructive sleep apnoea: a randomised prospective parallel trial. Lancet 1999;353(9170):2100-2105.

32. Redline S, Adams N, Strauss ME, Roebuck T, Winters M, Rosenberg C. Improvement of mild sleep-disordered breathing with CPAP compared with conservative therapy. Am J Respir Crit Care Med 1998;157(3 Pt 1):858-865.

33. Zimmerman ME, Arnedt JT, Stanchina M, Millman RP, Aloia MS. Normalization of memory performance and positive airway pressure 


\section{AdHEREnCE In SubJects With OSA Receiving CPAP}

adherence in memory-impaired patients with obstructive sleep apnea. Chest 2006;130(6):1772-1778.

34. Aloia MS, Knoepke CE, Lee-Chiong T. The new local coverage determination criteria for adherence to positive airway pressure treatment: testing the limits? Chest 2010;138(4):875-879.

35. Wickwire EM, Lettieri CJ, Cairns AA, Collop NA. Maximizing positive airway pressure adherence in adults: a common-sense approach. Chest 2013;144(2):680-693.

36. Brown LK. Use it or lose it: Medicare's new paradigm for durable medical equipment coverage? Chest 2010;138(4):785-789.

37. Romero E, Krakow B, Haynes P, Ulibarri V. Nocturia and snoring: predictive symptoms for obstructive sleep apnea. Sleep Breath 2010; 14(4):337-343.

38. Krakow B, Ulibarri VA, Romero EA, McIver ND. A two-year prospective study on the frequency and co-occurrence of insomnia and sleep-disordered breathing symptoms in a primary care population. Sleep Med 2013;14(9):814-823.

39. Fitzgerald MP, Mulligan M, Parthasarathy S. Nocturic frequency is related to severity of obstructive sleep apnea, improves with continuous positive airways treatment. Am J Obstet Gynecol 2006;194(5): 1399-1403.

40. Bliwise DL, Friedman L, Hernandez B, Zeitzer JM, Kushida CA, Yesavage JA. Nocturia reported in nightly sleep diaries: common occurrence with significant implications? Health Psychol 2014; 33(11):1362-1365.

41. Krakow B, Melendrez D, Ferreira E, Clark J, Warner TD, Sisley B, Sklar D. Prevalence of insomnia symptoms in patients with sleepdisordered breathing. Chest 2001;120(6):1923-1929.

42. Smith S, Sullivan K, Hopkins W, Douglas J. Frequency of insomnia report in patients with obstructive sleep apnoea hypopnea syndrome (OSAHS). Sleep Med 2004;5(5):449-456.

43. Wickwire EM, Collop NA. Insomnia and sleep-related breathing disorders. Chest 2010;137(6):1449-1463.

44. American Academy of Sleep Medicine. The International classification of sleep disorders: diagnostic and coding manual. 2nd edition. Westchester, Illinois: American Academy of Sleep Medicine 2005: 51-55.

45. Bastien $\mathrm{CH}$, Vallières $\mathrm{A}$, Morin $\mathrm{CM}$. Validation of the Insomnia Severity Index as an outcome measure for insomnia research. Sleep Med 2001;2(4):297-307.

46. Johns MW. A new method for measuring daytime sleepiness: the Epworth sleepiness scale. Sleep 1991;14(6):540-545.

47. Berry RB, Budhiraja R, Gottlieb DJ, Gozal D, Iber C, Kapur VK, et al. Rules for scoring respiratory events in sleep: update of the 2007 AASM Manual for the Scoring of Sleep and Associated Events: deliberations of the Sleep Apnea Definitions Task Force of the American Academy of Sleep Medicine. J Clin Sleep Med 2012;8(5):597619.
48. Kushida CA, Littner MR, Hirshkowitz M, Morgenthaler TI, Alessi CA, Bailey D, et al. Practice parameters for the use of continuous and bilevel positive airway pressure devices to treat adult patients with sleep-related breathing disorders. Sleep 2006;29(3):375-380.

49. Falcone VA, Damiani MF, Quaranta VN, Capozzolo A, Resta O. Polysomnograph chart view by patients: a new educational strategy to improve CPAP adherence in sleep apnea therapy. Respir Care 2014;59(2):193-198

50. Nadeem R, Rishi MA, Srinivasan L, Copur AS, Naseem J. Effect of visualization of raw graphic polysomnography data by sleep apnea patients on adherence to CPAP therapy. Respir Care 2013;58(4):607613.

51. Patel N, Sam A, Valentin A, Quan SF, Parthasarathy S. Refill rates of accessories for positive airway pressure therapy as a surrogate measure of long-term adherence. J Clin Sleep Med 2012;8(2):169175 .

52. Asghari A, Mohammadi F, Kamrava SK, Tavakoli S, Farhadi M. Severity of depression and anxiety in obstructive sleep apnea syndrome. Eur Arch Otorhinolaryngol 2012;269(12):2549-2553.

53. Babson KA, Del Re AC, Bonn-Miller MO, Woodward SH. The comorbidity of sleep apnea and mood, anxiety, and substance use disorders among obese military veterans within the Veterans Health Administration. J Clin Sleep Med 2013;9(12):1253-1258.

54. Björnsdóttir E, Benediktsdóttir B, Pack AI, Arnardottir ES, Kuna ST, Gíslason $\mathrm{T}$, et al. The prevalence of depression among untreated obstructive sleep apnea patients using a standardized psychiatric interview. J Clin Sleep Med 2016;12(1):105-112.

55. Harris M, Glozier N, Ratnavadivel R, Grunstein RR. Obstructive sleep apnea and depression. Sleep Med Rev 2009;13(6):437-444.

56. Lee K, Baron K, Soca R, Attarian H. The prevalence and characteristics of REM sleep without atonia (RSWA) in patients taking antidepressants. J Clin Sleep Med 2015 [Epub ahead of print].

57. Sharafkhaneh A, Giray N, Richardson P, Young T, Hirshkowitz M. Association of psychiatric disorders and sleep apnea in a large cohort. Sleep 2005;28(11):1405-1411.

58. Broström A, Strömberg A, Mårtensson J, Ulander M, Harder L, Svanborg E. Association of Type D personality to perceived side effects and adherence in CPAP-treated patients with OSAS. J Sleep Res 2007;16(4):439-447.

59. Dunn RL, Donoghue JM, Ozminkowski RJ, Stephenson D, Hylan TR. Longitudinal patterns of antidepressant prescribing in primary care in the UK: comparison with treatment guidelines. J Psychopharmacol 1999;13(2):136-143.

60. McFadyen TA, Espie CA, McArdle N, Douglas NJ, Engleman HM. Controlled, prospective trial of psychosocial function before and after continuous positive airway pressure therapy. Eur Respir J 2001;18(6):996-1002.

This article is approved for Continuing Respiratory Care Education credit. For information and to obtain your $\mathrm{CRCE}$

(free to AARC members) visit www.rcjournal.com

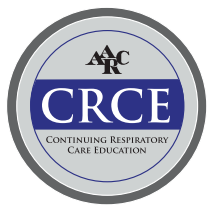

